2009-01-01

\title{
Imaging Reconstruction for Light Scattering from a Tenuous Random Medium
}

Jonathan Blackledge

Technological University Dublin, jonathan.blackledge@tudublin.ie

Follow this and additional works at: https://arrow.tudublin.ie/engscheleart

Part of the Optics Commons, and the Signal Processing Commons

\section{Recommended Citation}

Imaging Reconstruction for Light Scattering from a Tenuous Random Medium, Blackledge, Jonathan; ISSC 2009, UCD, June 10-11th 2009 , University College Dublin, 2009

This Conference Paper is brought to you for free and open access by the School of Electrical and Electronic Engineering at ARROW@TU Dublin. It has been accepted for inclusion in Conference papers by an authorized administrator of ARROW@TU Dublin. For more information, please contact arrow.admin@tudublin.ie, aisling.coyne@tudublin.ie,gerard.connolly@tudublin.ie.

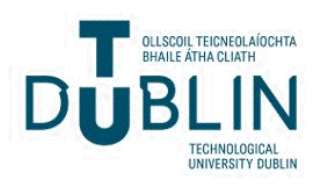




\title{
Imaging Reconstruction for Light Scattering from a Tenuous Random Medium
}

\author{
Jonathan Blackledge
}

\author{
SFI Stokes Professor of DSP \\ School of Electrical Engineering Systems \\ Faculty of Engineering \\ Dublin Institute of Technology \\ E-mail: jonathan.blackledge@dit.ie
}

\begin{abstract}
We consider the basis for describing strong scattering in terms of diffusive processes based on the diffusion equation. Intermediate strength scattering is then considered in terms of a fractional diffusion equation which is studied using results from fractional calculus. This approach is justified in terms of the generalization of a random walk model with no statistical bias in the phase to a random walk that has a phase bias and is thus, only 'partially' or 'fractionally' diffusive. A Green's function solution to the fractional diffusion equation is studied and a result derived that provides a model for an incoherent image generated by light scattering from a tenuous random medium. Applications include image enhancement of star fields and other cosmological bodies imaged through interstellar dust clouds. An example of this application is given.
\end{abstract}

Keywords - Tenuous Random Media, Fractional Diffusion, Image Processing

\section{INTRODUCTION}

A conventional approach to modelling light scattering in random media is to consider the scatterer to be a stochastic function with a characteristic Probability Density Function (PDF) under the weak scattering approximation [1]. In the far field, the scattering amplitude is then given by the Fourier transform of the scattering function and the intensity of the scattered field (i.e. the measurable quantity, at optical frequencies and above) is determined by the Fourier transform of the autocorrelation of the scattering function. The inverse scattering problem is then reduced to estimating the correlation function by Fourier inversion and then solving the phase reconstruction problem to recover the scattering function from its autocorrelation function.

Multiple scattering processes are often modelled using a statistical approach [2]. The aim is to developed a model of the PDF for the scattered field itself rather than for the scattering function. This involves concepts traditionally associated with the kinetic theory of gases in which the random motion of particles undergoing elastic collisions and following 'random walks' is 'replaced' with the random scattering of an electric field, for example, from multiple scattering sites. The total contribution of the multiple scattering process after $N$ scattering interactions is given by [3]

$$
E=\sum_{j=1}^{N} a_{j} \exp \left(i \theta_{j}\right)
$$

where the amplitude $a_{j}$, the phase $\theta_{j}$ and $N$ are independent random variables. While this approach provides physically informative models for the PDF that can be used for the statistical characterisation of an image and image segmentation (using a moving 'window') to locate statistically significant features, it does not directly help in the development of algorithms for image restoration and reconstruction [4]. On the other hand, random walk models provide the physical basis for diffusion processes in general. This is the essential 'link' to modelling multiple scattering processes in terms of solutions to the diffusion equation for the intensity of light. 
In certain circumstances, multiple scattering may only involve a small number of interactions. This occurs when light interacts with tenuous media, for example, and is considered to be one of the most difficult scenarios to model accurately. Diffusion processes are not applicable in such cases. In this paper, we study an approach to solving this problem using the fractional diffusion equation.

\section{Optical DifFusion}

When light is scattered by one localized centre, the single or 'weak' scattering approximation can be used, i.e. the Born approximation [1]. However, when these centres are grouped together, multiple light scattering occurs. The randomness of multiple interactions tends to be averaged out by the large number of scattering events that occur leading to a deterministic distribution of intensity. This is exemplified by a light beam propagating through thick fog, for example. In this sense, multiple scattering is highly analogous to diffusion, and the terms multiple scattering and diffusion are interchangeable in many contexts. Optical elements designed to produce multiple scattering are thus known as diffusers. The diffusion equation can then be used to model such systems in the same way as it is used to model temperature distributions or particle concentrations, for example, and any system that is the result of a large ensemble of particles or waves undergoing random elastic collisions or scattering interactions respectively.

Suppose we consider the three-dimensional diffusion of light to be based on a three-dimensional random walk. Each scattering event is taken to be a point of the random walk in which a ray of light changes its direction randomly (any direction between 0 and $4 \pi$ radians). The light field is taken to be composed of a complex of rays, each of which propagates through the diffuser in a way that is incoherent and uncorrelated in time. If this is the case, then the propagation of light can be considered to be analogous to a process of (classical) diffusion. Instead of modelling the process in terms of the three-dimensional inhomogeneous wave equation (for the a spatially variable wavespeed $c(\mathbf{r}), \mathbf{r}=\hat{\mathbf{x}} x+\hat{\mathbf{y}} y+\hat{\mathbf{z}} z$ with PDF $\operatorname{Pr}[c(\mathbf{r})])$

$$
\left(\nabla^{2}-\frac{1}{c^{2}(\mathbf{r})} \frac{\partial^{2}}{\partial t^{2}}\right) u(\mathbf{r}, t)=0
$$

with light intensity given by $I=|u|^{2}$, we consider the intensity to be given by the solution of the homogeneous diffusion equation

$$
\left(\nabla^{2}-\frac{1}{D} \frac{\partial}{\partial t}\right) I(\mathbf{r}, t)=0
$$

with initial condition $I(\mathbf{r}, t)=I_{0}(\mathbf{r})$ at $t=0$. This model assumes that the diffusivity $D$ is constant throughout the diffuser which is equivalent to a random scattering model (based on a solution to the wave equation) in which $\operatorname{Pr}[c(\mathbf{r})]$ is constant throughout the diffuser, i.e. stationary statistics.

In multiple wave scattering theory, we consider a wavefront travelling through space and scattering from multiple interaction sites, each of which changes the direction of propagation in an entirely random way with no directional bias over $4 \pi$ radian. The mean free path is taken to be the average number of wavelengths required for the wavefront to propagate from one interaction to another as described by the free space Green's function. After scattering from many sites, the wavefront can be considered to have diffused through the 'diffuser'. Here, the mean free path is a measure of the density of scattering sites, which in turn, is a measure of the diffusivity of the medium $D$. As $D \rightarrow \infty$, the medium becomes increasingly tenuous.

\section{Optical Diffusion Equation}

Consider the three-dimensional homogeneous time dependent wave equation

$$
\nabla^{2} u-\frac{1}{c_{0}^{2}} \frac{\partial^{2}}{\partial t^{2}} u=0
$$

where $c_{0}$ is taken to be a constant (light speed). Let

$$
u(x, y, z, t)=\phi(x, y, z, t) \exp (i \omega t)
$$

where it is assumed that field $\phi$ varies significantly slowly in time compared with $\exp (i \omega t)$ and note that

$$
u^{*}(x, y, z, t)=\phi^{*}(x, y, z, t) \exp (-i \omega t)
$$

is also a solution to the wave equation. Differentiating

$$
\nabla^{2} u=\exp (i \omega t) \nabla^{2} \phi
$$

and

$$
\begin{aligned}
\frac{\partial^{2}}{\partial t^{2}} u & =\exp (i \omega t)\left(\frac{\partial^{2}}{\partial t^{2}} \phi+2 i \omega \frac{\partial \phi}{\partial t}-\omega^{2} \phi\right) \\
& \simeq \exp (i \omega t)\left(2 i \omega \frac{\partial \phi}{\partial t}-\omega^{2} \phi\right)
\end{aligned}
$$

when

$$
\left|\frac{\partial^{2} \phi}{\partial t^{2}}\right|<<2 \omega\left|\frac{\partial \phi}{\partial t}\right| .
$$

Under this condition, the wave equation reduces to

$$
\left(\nabla^{2}+k^{2}\right) \phi=\frac{2 i k}{c_{0}} \frac{\partial \phi}{\partial t}
$$

where $k=\omega / c_{0}$. However, since $u^{*}$ is also a solution,

$$
\left(\nabla^{2}+k^{2}\right) \phi^{*}=-\frac{2 i k}{c_{0}} \frac{\partial \phi^{*}}{\partial t}
$$


and thus,

$$
\phi^{*} \nabla^{2} \phi-\phi \nabla^{2} \phi^{*}=\frac{2 i k}{c_{0}}\left(\phi^{*} \frac{\partial \phi}{\partial t}+\phi \frac{\partial \phi^{*}}{\partial t}\right)
$$

which can be written in the form

$$
\nabla^{2} I-2 \nabla \cdot\left(\phi \nabla \phi^{*}\right)=\frac{2 i k}{c_{0}} \frac{\partial I}{\partial t}
$$

where $I=\phi \phi^{*}=|\phi|^{2}$. Let $\phi$ be given by

$$
\phi(\mathbf{r}, t)=A(\mathbf{r}, t) \exp (i k \hat{\mathbf{n}} \cdot \mathbf{r})
$$

where $\hat{\mathbf{n}}$ is a unit vector and $A$ is the amplitude function. Differentiating, and noting that $I=A^{2}$, we obtain

$$
\hat{\mathbf{n}} \cdot \nabla A=\frac{2}{c_{0}} \frac{\partial A}{\partial t}
$$

or

$$
\left(\frac{\partial}{\partial x}+\frac{\partial}{\partial y}+\frac{\partial}{\partial z}\right) A(x, y, z, t)=\frac{2}{c_{0}} \frac{\partial}{\partial t} A(x, y, z, t)
$$

which is the unconditional continuity equation for the amplitude $A$ of a wavefield

$$
u(\mathbf{r}, t)=A(\mathbf{r}, t) \exp [i(k \hat{\mathbf{n}} \cdot \mathbf{r}+\omega t)]
$$

where $A$ varies slowly with time.

The equation

$$
\nabla^{2} I-2 \nabla \cdot\left(\phi \nabla \phi^{*}\right)=\frac{2 i k}{c_{0}} \frac{\partial I}{\partial t}
$$

is valid for $k=k_{0}-i \kappa$ (i.e. $\omega=\omega_{0}-i \kappa c_{0}$ ) and so, by equating the real and imaginary parts, we have

$$
D \nabla^{2} I+2 \operatorname{Re}\left[\nabla \cdot\left(\phi \nabla \phi^{*}\right)\right]=\frac{\partial I}{\partial t}
$$

and

$$
\operatorname{Im}\left[\nabla \cdot\left(\phi \nabla \phi^{*}\right)\right]=-\frac{k_{0}}{c_{0}} \frac{\partial I}{\partial t}
$$

respectively where $D=c_{0} / 2 \kappa$, so that under the condition

$$
\operatorname{Re}\left[\nabla \cdot\left(\phi \nabla \phi^{*}\right)\right]=0
$$

we obtain

$$
D \nabla^{2} I=\frac{\partial I}{\partial t} .
$$

This is the diffusion equation for the intensity of light $I$. The condition required to obtain this result can be justified by applying a boundary condition on the surface $S$ of a volume $V$ over which the equation is taken to conform. Using the divergence theorem

$$
\begin{gathered}
\operatorname{Re} \int_{V} \nabla \cdot\left(\phi \nabla \phi^{*}\right) d^{3} \mathbf{r}=\operatorname{Re} \oint_{S} \phi \nabla \phi^{*} \cdot \hat{\mathbf{n}} d^{2} \mathbf{r} \\
=\oint_{S}\left(\phi_{r} \nabla \phi_{r}+\phi_{i} \nabla \phi_{i}\right) \cdot \hat{\mathbf{n}} d^{2} \mathbf{r}
\end{gathered}
$$

and if

$$
\phi_{r}(\mathbf{r}, t) \nabla \phi_{r}(\mathbf{r}, t)=-\phi_{i}(\mathbf{r}, t) \nabla \phi_{i}(\mathbf{r}, t), \quad \mathbf{r} \in S
$$

then the surface integral is zero and

$$
D \nabla^{2} I(\mathbf{r}, t)=\frac{\partial}{\partial t} I(\mathbf{r}, t), \quad \mathbf{r} \in V .
$$

This boundary condition can be written as

$$
\frac{\nabla \phi_{r}}{\nabla \phi_{i}}=-\tan \theta
$$

where $\theta$ is the phase of the field $\phi$ which implies that the amplitude $A$ of $\phi$ is constant on the boundary (i.e. $A(\mathbf{r}, t)=A_{0}, \quad \mathbf{r} \in S, \quad \forall t$ ), since

$$
\begin{gathered}
\frac{\nabla A_{0} \cos \theta(\mathbf{r}, t)}{\nabla A_{0} \sin \theta(\mathbf{r}, t)}=-\frac{A_{0} \sin \theta(\mathbf{r}, t) \nabla \theta(\mathbf{r}, t)}{A_{0} \cos \theta(\mathbf{r}, t) \nabla \theta(\mathbf{r}, t)} \\
=-\tan \theta(\mathbf{r}, t), \quad \mathbf{r} \in S .
\end{gathered}
$$

\section{a) Diffused Image Equation}

Suppose we record the intensity $I$ of a light field in the $x y$-plane for a fixed value of $z$. Then for $z=z_{0}$ say

$$
I(x, y, t) \equiv I\left(x, y, z_{0}, t\right)
$$

so that

$$
\frac{\partial}{\partial t} I(x, y, t)=D \nabla^{2} I(x, y, t) .
$$

Let this two-dimensional diffusion equation be subject to the initial condition

$$
I(x, y, 0)=I_{0}(x, y) .
$$

Then, at any time $T>0$, it can be assumed that light diffusion is responsible for generating image $I$ and that as time increases, the image becomes progressively more diffused, the solution being given by, for the infinite domain and ignoring scaling [6]

$$
I(x, y, T)=\exp \left[-\left(\frac{x^{2}+y^{2}}{4 D T}\right)\right] \otimes_{2} I_{0}(x, y)
$$

where $\otimes_{2}$ denotes the two-dimensional convolution integral.

b) Inverse Solution

If we record an image at a time $t=T$ then by Taylor expanding $I$ at $t=0$ we can write

$$
\begin{gathered}
I(x, y, 0)=I(x, y, T) \\
+\sum_{n=1}^{\infty} \frac{(-1)^{n}}{n !} T^{n}\left[\frac{\partial^{n}}{\partial t^{n}} I(x, y, t)\right]_{t=T} .
\end{gathered}
$$

From the diffusion equation

$$
\frac{\partial^{2} I}{\partial t^{2}}=D \nabla^{2} \frac{\partial I}{\partial t}=D^{2} \nabla^{4} I
$$




$$
\frac{\partial^{3} I}{\partial t^{3}}=D \nabla^{2} \frac{\partial^{2} I}{\partial t^{2}}=D^{3} \nabla^{6} I
$$

so that, by induction, we can write

$$
\left[\frac{\partial^{n}}{\partial t^{n}} I(x, y, t)\right]_{t=T}=D^{n} \nabla^{2 n} I(x, y, T) .
$$

Substituting this result into the series for $I(x, y, 0) \equiv I_{0}(x, y)$, we get

$$
\begin{gathered}
I_{0}(x, y)=I(x, y, T) \\
+\sum_{n=1}^{\infty} \frac{(-1)^{n}}{n !}(D T)^{n} \nabla^{2 n} I(x, y, T)
\end{gathered}
$$

The 'high emphasis filter' [4] is then obtained when $D T<<1$, i.e.

$$
I_{0}(x, y) \sim I(x, y, T)-D T \nabla^{2} I(x, y, T) .
$$

\section{Fractional Diffusion}

The diffusion equation models a macroscopic field which is the result of an ensemble of incoherent random walks characterised by a $\sqrt{t}$ scaling law. Hurst processes, describe random walks that have a directional bias and are characterised by the scaling law $t^{H}, H \in(0.5,1]$ [7], [8]. As the value of $H$ approaches 1, the processes become increasingly persistent. In terms of the multiple scattering of light from a random medium, increasing persistence relates to multiple scattering from fewer sites so that the light path has a greater directional bias. We consider the characterisation of this by generalizing the diffusion operator

$$
\nabla^{2}-\sigma \frac{\partial}{\partial t}
$$

to the fractional form [9], [10]

$$
\nabla^{2}-\sigma^{q} \frac{\partial^{q}}{\partial t^{q}}
$$

where $q \in[1,2]$ and $D^{q}=1 / \sigma^{q}$ is the fractional diffusivity. Fractional diffusive processes can therefore be interpreted as intermediate between diffusive processes proper (random phase walks with $H=0.5$; diffusive processes with $q=1$ ) and 'propagative process' (coherent phase walks for $H=1$; propagative processes with $q=2$ ). It should be noted that the fractional diffusion operator given above is the result of a phenomenology. It is a generalisation of a well known differential operator to fractional form which follows from a physical analysis of a fully incoherent random process and its generalisation to fractional form in terms of the Hurst exponent $H$. The solution to fractional partial differential equations of this type requires application of the fractional calculus [11] - [15] which is considered in the following section.

\section{Fractional Diffusion Equation}

Consider the two-dimensional fractional diffusion equation for the intensity $I(x, y, t)$ of light in the image plane located at $z$ given by

$$
\nabla^{2} I(\mathbf{r}, t)-\sigma^{q} \frac{\partial^{q}}{\partial t^{q}} I(\mathbf{r}, t)=I_{0}(\mathbf{r}, t)
$$

where $\mathbf{r}=\hat{\mathbf{x}} x+\hat{\mathbf{y}} y, r \equiv|\mathbf{r}|$ and $I_{0}(\mathbf{r})$ is now a (twodimensional) source function. Using the Fourier based operator for a fractional derivative, we can transform this equation into the form

$$
\left(\nabla^{2}+\Omega_{q}^{2}\right) \widetilde{I}(\mathbf{r}, \omega)=\widetilde{I}_{0}(\mathbf{r}, \omega)
$$

where

$$
\begin{gathered}
\widetilde{I}(\mathbf{r}, \omega)=\int_{-\infty}^{\infty} I(\mathbf{r}, t) \exp (-i \omega t) d t \\
\widetilde{I}_{0}(\mathbf{r}, \omega)=\int_{-\infty}^{\infty} I_{0}(\mathbf{r}, t) \exp (-i \omega t) d t
\end{gathered}
$$

and

$$
\Omega_{q}^{2}=-(i \omega \sigma)^{q}, \quad \Omega_{q}= \pm i(i \omega \sigma)^{q / 2} .
$$

The Green's function solution for this equation (in the infinite domain) is

$$
\widetilde{I}(\mathbf{r}, \omega)=g(r, \omega) \otimes_{2} \widetilde{I}_{0}(\mathbf{r}, \omega)
$$

where $g$ is the 'outgoing' Green function given by (for $\left|\Omega_{q} r\right|>>1$ and ignoring scaling) [6]

$$
g(r, \omega) \simeq \frac{\exp \left(i \Omega_{q} r\right)}{\sqrt{\Omega_{q} r}} .
$$

For $\Omega_{q}=i(i \omega \sigma)^{q / 2}$, Fourier inversion, yields the time dependent Green's function (obtained by writing the exponential function in its series form).

$$
\begin{aligned}
& G(r, t)=\frac{1}{\sqrt{r}} \frac{1}{\sigma^{q / 4} t^{1-q / 4}}-\sqrt{r} \sigma^{q / 4} \delta^{q / 4}(t) \\
& +\sum_{n=1}^{\infty} \frac{(-1)^{n+1}}{(n+1) !} r^{(2 n+1) / 2} \sigma^{3 n q / 4} \delta^{3 n q / 4}(t),
\end{aligned}
$$

the solution for $I(\mathbf{r}, t)$ being given by

$$
I(\mathbf{r}, t)=G(r, t) \otimes_{2} \otimes_{t} I_{0}(\mathbf{r}, t)
$$

where $\otimes_{t}$ denotes the (non-causal) convolution integral over time $t$. Simplification of this infinite sum representation for $G$ can be addressed be considering suitable asymptotics, the most significant of which (for arbitrary values of $r$ ) is the case when the (fractional) diffusivity $D$ is large. In particular, we note that as $\sigma \rightarrow 0$,

$$
G(r, t)=\frac{1}{\sqrt{r} \sigma^{q / 4} t^{1-q / 4}} .
$$


Thus, we can consider a solution to the twodimensional fractional diffusion equation (for a tenuous medium when $D \rightarrow \infty$ ) of the form (at any time $t=T$ and ignoring scaling)

$$
I(x, y)=\frac{1}{\left(x^{2}+y^{2}\right)^{\frac{1}{4}}} \otimes_{2} I_{0}(x, y) .
$$

\section{Deconvolution}

In the presence of additive noise $n(x, y)$, the deconvolution problem is as follows: Given that

$$
I(x, y)=p(x, y) \otimes_{2} I_{0}(x, y)+n(x, y)
$$

where $\operatorname{Pr}[n(x, y)]$ is known (ideally), find an estimate for $I_{0}$. This is a common problem in optics (digital image processing) known as the deconvolution problem whose solution is fundamental to image restoration and reconstruction [4], [5]. In terms of the material presented in this paper, there are two Point Spread Functions (PSF) $p(x, y)$ that have been considered: For full diffusion (strong scattering)

$$
p(x, y)=\exp \left[-\left(\frac{x^{2}+y^{2}}{4 D T}\right)\right]
$$

and for fractional diffusion (intermediate scattering in a tenuous medium with large diffusivity)

$$
p(x, y)=\frac{1}{\left(x^{2}+y^{2}\right)^{\frac{1}{4}}} .
$$

We note that (ignoring scaling)

$$
\exp \left[-\left(\frac{x^{2}+y^{2}}{4 D T}\right)\right] \leftrightarrow \exp \left[-4 D T\left(k_{x}^{2}+k_{y}^{2}\right)\right]
$$

and $[8]$

$$
\frac{1}{\left(x^{2}+y^{2}\right)^{\frac{1}{4}}} \leftrightarrow \frac{1}{\left(k_{x}^{2}+k_{y}^{2}\right)^{\frac{3}{4}}}
$$

where $\leftrightarrow$ denotes transformation from real space to Fourier space. In the latter case, the filter is a 'fractal filter' and thus, if $I_{0}$ is characterised by white noise, then the output $I$ is a Mandelbrot surface with a fractal dimension of 2.5 [8]. In the absence of noise, the inverse solution for $I_{0}$ can be written in the form

$$
I_{0}(x, y)=\nabla^{\frac{3}{2}} I(x, y),
$$

a result that is based on the application of the fractional Laplacian or Riesz operator [8]

$$
\nabla^{q} \leftrightarrow\left(k_{x}^{2}+k_{y}^{2}\right)^{\frac{q}{2}}
$$

There are a range of approaches to solving the one-dimensional and two-dimensional deconvolution problem in practice (i.e. with additive noise) leading to the classification of different 'inverse filters' (e.g. [4], [5]). If a priori information on the statistics of the noise function $n$ and the object function $I_{0}$ is available, then Bayesian estimation methods are preferable in the design of filters whose performance then depends on statistical parameters such as the standard deviation. In some cases, an estimate of $\operatorname{Pr}[n(x, y)]$ can be obtained by taking an image (or a number of images to obtain a statistically significant result) with zero input, i.e. with $I_{0}=0$. This provides a method of validating an idealised PDF through data fitting and, thus, determination of the statistical parameters from which a theoretical PDF is composed. In cases when experimental determinism is not practically possible, statistical models must be utilized [3]. However, with regard to incoherent imaging systems, the noise function tends to be Gaussian distributed - a result of the noise being a linear combination of many different independent noise sources which combine to produce Gaussian noise (a consequence of the Central Limit Theorem).

Using Bayes rule, the aim is to find an estimate for $I_{0}$ such that

$$
\frac{\partial}{\partial I_{0}} \ln \operatorname{Pr}[n(x, y)]+\frac{\partial}{\partial I_{0}} \ln \operatorname{Pr}\left[I_{0}(x, y)\right]=0 .
$$

Consider the following models for the PDFs: (i) A Gaussian distribution for the noise (ignoring scaling and where $\sigma_{n}^{2}$ is the standard deviation of $n$ )

$$
\begin{gathered}
\operatorname{Pr}[n(x, y)]= \\
\exp \left(-\frac{1}{\sigma_{n}^{2}} \iint\left[\left(I(x, y)-p(x, y) \otimes_{2} I_{0}(x, y)\right]^{2} d x d y\right) .\right.
\end{gathered}
$$

(ii) A Gaussian distribution for the object function (ignoring scaling and where $\sigma_{I_{0}}^{2}$ is the standard deviation of $I_{0}$ )

$$
\operatorname{Pr}\left[I_{0}(x, y)\right]=\exp \left(-\frac{1}{\sigma_{I_{0}}^{2}} \iint I_{0}^{2}(x, y) d x d y\right) .
$$

Differentiating, these statistical models yield the equation

$$
\begin{gathered}
I(x, y) \odot_{2} p(x, y) \\
=\frac{\sigma_{n}^{2}}{\sigma_{I_{0}}^{2}} I_{0}(x, y)+\left[p(x, y) \otimes_{2} I_{0}(x, y)\right] \odot_{2} p(x, y)
\end{gathered}
$$

where $\odot_{2}$ denotes the two-dimensional correlation integral. In Fourier space, this equation becomes

$$
\begin{gathered}
\widetilde{I}\left(k_{x}, k_{y}\right) P^{*}\left(k_{x}, k_{y}\right) \\
=\frac{\sigma_{n}^{2}}{\sigma_{I_{0}}^{2}} \widetilde{I}_{0}\left(k_{x}, k_{y}\right)+\left|P\left(k_{x}, k_{y}\right)\right|^{2} I_{0}\left(k_{x}, k_{y}\right) .
\end{gathered}
$$

The Bayesian a Posteriori filter $F\left(k_{x}, k_{y}\right)$ (for Gaussian statistics) is then given by

$$
F\left(k_{x}, k_{y}\right)=\frac{P^{*}\left(k_{x}, k_{y}\right)}{\left|P\left(k_{x}, k_{y}\right)\right|^{2}+\sigma_{n}^{2} / \sigma_{I_{0}}^{2}}
$$

where $\sigma_{I_{0} / \sigma_{n}}$ defines the signal-to-noise ratio of $I(x, y)$ and $\widetilde{I}_{0}\left(k_{x}, k_{y}\right)=F\left(k_{x}, k_{y}\right) \widetilde{I}\left(k_{x}, k_{y}\right)$. 


\section{Example Application}

Fractional diffusion models apply to scattering processes that occur in a tenuous and extremely rarefied medium. In applied optics, one of the most common examples of this phenomena occurs in astronomy and the processes associated with light scattering from interstellar dust which consists of an extremely dilute (by terrestrial standards) mixture of ions, atoms, molecules, and larger dust grains, consisting of about $99 \%$ gas and $1 \%$ dust by mass. Densities range from a few thousand to a few hundred million particles per cubic meter with an average value in the Milky Way Galaxy, for example, of a million particles per cubic meter. In comparison with the scattering of light from earthbased random media, for example, the interstellar medium is highly diffuse and therefore ideal for applying light scattering models based on fractional diffusion when $D \rightarrow \infty$.

Figure 1 shows the application of the filter given by equation (1) with

$$
P\left(k_{x}, k_{y}\right)=\frac{1}{\left(k_{x}^{2}+k_{y}^{2}\right)^{0.75}}
$$

for an optical image obtained from Stardust in Perseus [16]. The image is part of the constellation of Perseus as observed through an interstellar dust cloud that covers nearly four degrees of the sky observed approximately $10^{3}$ light-years away.

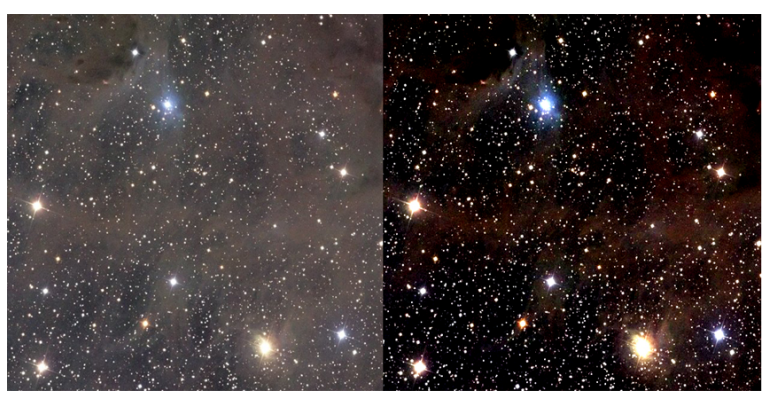

Fig. 1: Fractional diffusion based deconvolution (right) for $\sigma_{n} / \sigma_{I_{0}}=1$ of a dust clouded star field (left) in the constellation of Pegasus.

\section{Conclusions}

The use of fully diffusive processes for modelling strong scattering provides a result that is applicable in solving the inverse (multiple) scattering problem. This requires the formulation of a deconvolution algorithm for a Gaussian PSF. We have extended this approach to model intermediate scattering by generalizing the homogeneous diffusion equation to fractional form for a fractional diffusivity $D$. An asymptotic solution has been considered based on the condition $D \rightarrow \infty$ which yields a characteristic filter of the form $\left(k_{x}^{2}+k_{y}^{2}\right)^{-0.75}$ in Fourier space. This filter is the transfer function associated with an optical system involving the intermediate strength scattering of light in a tenuous medium.

\section{REFERENCES}

[1] J, M. Blackledge, "Digital Image processing", Horwood Publishing, 2006.

[2] P. A. Martin, "Multiple Scattering: Interaction of Time-Harmonic Waves with N Obstacles", Encyclopedia of Mathematics and its Applications 10\%, Cambridge University Press, 2006.

[3] T. R. Field, "Electromagnetic Scattering from Random Media", International Series of Monographs on Physics - 144 Oxford Science Publications, 2009.

[4] R. H. T. Bates and M. J. McDonnal, "Image Restoration and Reconstruction", Oxford Science Publications, 1986.

[5] M. Bertero and B. Boccacci, "Introduction to Inverse Problems in Imaging", Institute of Physics Publishing, 1998.

[6] G. A. Evans, J. M. Blackledge and P. Yardley, "Analytical Solutions to Partial Differential Equations", Springer-Verlag, 2000.

[7] E. H. Hurst, "A Short Account of the Nile Basin", Cairo, Government Press, 1944.

[8] M. J. Turner, J. M. Blackledge and P. Andrews, "Fractal Geometry in Digital Imaging", Academic Press, 1997.

[9] R. Hilfer, "Foundations of Fractional Dynamics", Fractals Vol. 3, No. 3, 549-556, 1995.

[10] R. Hilfer, "Exact Solutions for a Class of Fractal Time Random Walks", Fractals, Vol. 3, No. 1, 211-216, 1995.

[11] K. B. Oldham and J. Spanier, "The Fractional Calculus", Academic Press, 1974.

[12] A. Dold and B. Eckmann (Eds.), "Fractional Calculus and its Applications", Springer, 1975.

[13] K. S. Miller and B. Ross, "An Introduction to the Fractional Calculus and Fractional Differential Equations", Wiley, 1993.

[14] S. G. Samko, A. Kilbas and O. I. Marichev, "Fractional Integrals and Derivatives: Theory and Applications", Gordon and Breach, 1993.

[15] V. Kiryakova, "Generalized Fractional Calculus and Applications", Longman, 1994.

[16] http://www.astronet.ru:8104/db/xware/msg/ 1224842 